\title{
Topology Processor design for EHV Substations
}

\author{
Wonkeun $\mathrm{Yu}^{1}$, Junghyun $\mathrm{Oh}^{2}$, Minhan $\mathrm{Yoon}^{3}$, Heungjae Lee ${ }^{3 *}$ \\ ${ }^{1}$ Department of Automatic Electrical Engineering Technology, Yeungnam University College, Daegu \\ 42415, Korea \\ ${ }^{2}$ Department of Robotics, Kwangwoon University, Seoul 01897, Korea \\ ${ }^{3}$ Department of Electrical Engineering, Kwangwoon University, Seoul 01897, Korea
}

\begin{abstract}
The intelligent application is one of the major issues in power system research. The topology identification is one of the most important modules to develop intelligent system such as fault diagnosis and restoration expert system. This paper presents the topology processor in order to identify topology effectively in a practical $765 \mathrm{kV}$ substation in Korea composed of the double-bus and $\mathbf{1 . 5}$ $\mathrm{CB}$, which is the typical structure of EHV substations. The proposed topology processor redefines switching group composed of circuit breaker, disconnecting switches and ground switches as aggregated circuit breaker. The proposed system remarkably reduces the search space and search time for topology identification. The proposed topology processor is developed using the data of a practical $765 \mathrm{kV}$ substation in Korea. The proposed system can be applied to intelligent systems such as fault diagnosis and restoration expert system and contribute to improving the performance of intelligent systems.
\end{abstract}

Keywords-Topology Processor, Topology Identification, Aggregated CB, 1.5CB.

\section{INTRODUCTION}

$\mathrm{T}$ OPOLOGY processor is a module to identify the real-time topology determined by the status of various switches such as circuit breaker, disconnecting switch and ground switch. The topology processor emulates the mechanism by which a power system expert identifies the connection structure of substation devices in the diagram. The topology processor is one of the most important modules to develop intelligent system such as fault diagnosis and restoration system. However, the topology identification is a very complicated problem. Up to now, the intelligent systems to be successfully developed does not exist much, which results from the topology identification rather than the complexity of domain knowledge.

Regarding topology processor, various papers have been reported. Ito[1] proposed the algorithm method to reconfigure a mathematical model about the change of a switch. Piereti[2] proposed the a new graph representation method named
Node-Depth Representation(NDR). He[3] proposed an efficient topology analysis algorithm to track the network connectivity for distribution system. Yehsakul[4] proposed the NCLU (Network Connectivity Local Update) algorithm using the tree. Lee[5,6] proposed the topology process in double-bus structured distribution substation.

This paper presents the topology processor in order to identify real-time topology effectively of a practical $765 \mathrm{kV}$ substation in Korea. A practical $765 \mathrm{kV}$ substation is the structure of double-bus and $1.5 \mathrm{CB}$ which is a typical structure for EHV(extra high voltage) substations. The devices of substation are connected complicatedly through the interlocked switching group composed of circuit breaker, disconnecting switches and ground switches. Under the complex connection structure of switches, topology identification is very difficult because a possible combination of switches increases exponentially.

The proposed topology processor used the aggregation method and reduced remarkably the search space for topology identification and enhanced the efficiency of identification. The proposed topology processor defines the switching group as an aggregated circuit breaker. The topology processor defines the connection structure between unit devices using the aggregated circuit breaker. As a result of aggregation method, the topology processor remarkably reduces the search space and search time for topology identification and enhances the performance.

The proposed topology processor is developed using the data of a practical $765 \mathrm{kV}$ substation in Korea.

\section{DEFINITIONS OF AGGREGATED CB}

The general structure of a practical $765 \mathrm{kV}$ substation in Korea shows in figure 1.

As shown in figure 1, a practical $765 \mathrm{kV}$ substation is composed of unit devices such as a few $765 \mathrm{kV}$ transmission lines(we define it as HTLs), $1.5 \mathrm{CB}$ structured $765 \mathrm{kV}$ double-bus(HBUSes), 2 to 4 of $765 \mathrm{kV} / 345 \mathrm{kV}$ transformer(MTRs), $\quad 1.5 \quad \mathrm{CB} \quad$ structured $345 \mathrm{kV}$ double-bus(LBUSes), a few $345 \mathrm{kV}$ transmission lines(LTLs), shunt reactor(SHRs) and switches like circuit breakers(CBs), disconnecting switches(DSs) and ground switches(ESs). The 


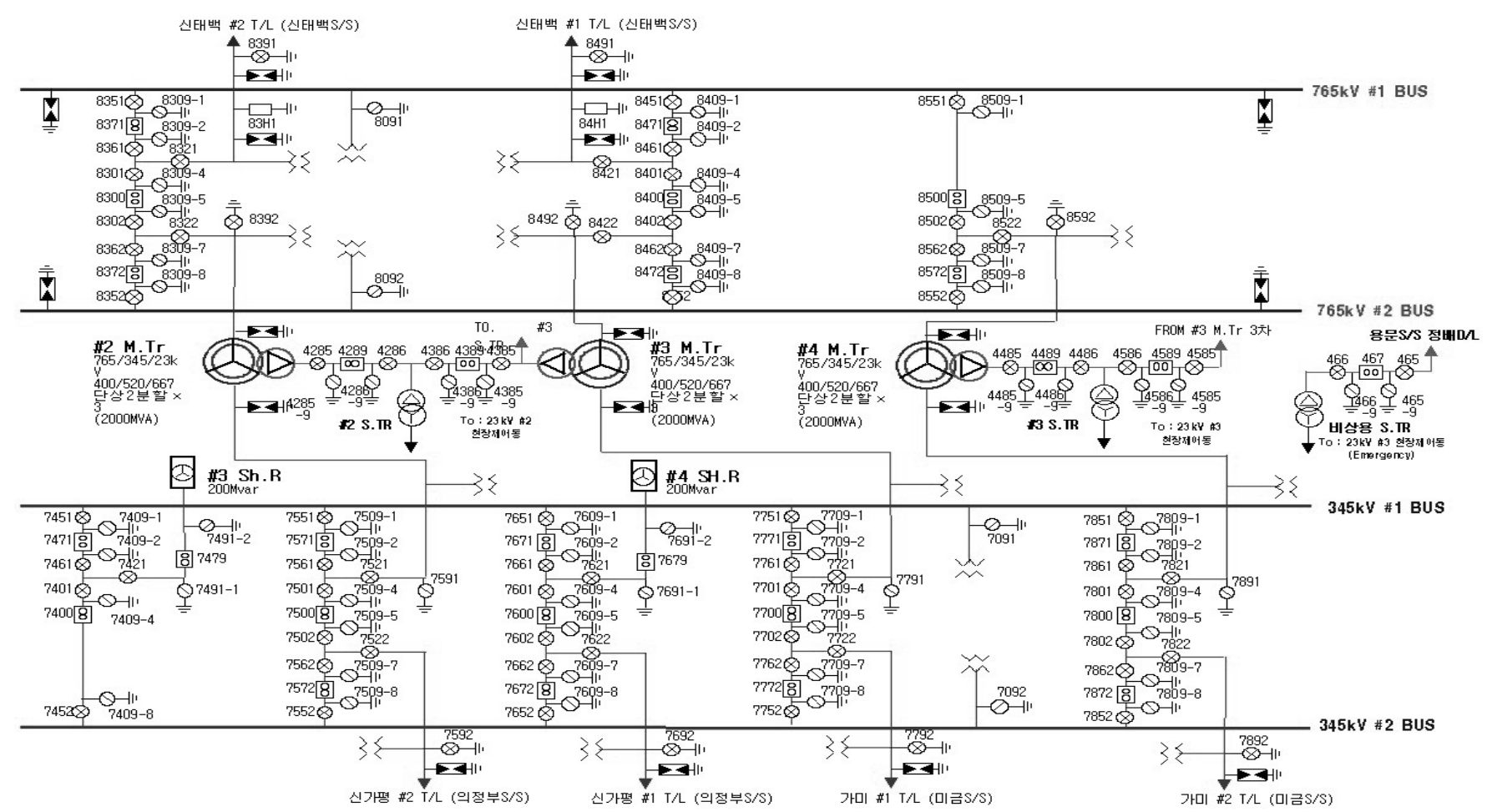

Fig. 1 Structure of a practical $765 \mathrm{kV}$ Substation

real-time topology of substation is determined by the ON/OFF status of circuit breaker and disconnecting switches.

As shown in figure 1, each unit devices are connected complicatedly through circuit breaker, disconnecting switch and ground switch. If these switches are used in topology processor, topology identification is very difficult because the possible combination of switches increases exponentially. This paper introduced the new aggregation technique to reduce the possible combination of switches.

Figure 2 shows the structure of switching group of $765 \mathrm{kV}$ substation.

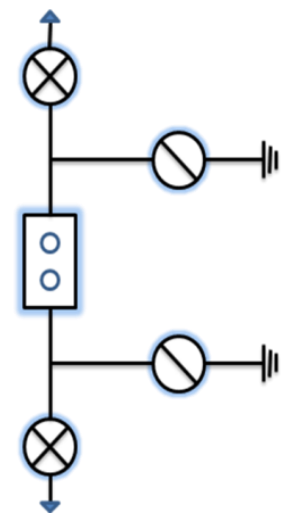

Fig. 2 Structure of switching group

As shown in figure 2, a circuit breaker composes a switching group with two series disconnecting switches and two parallel ground switches.

The switches of switching group are interlocked. This paper defines newly a switching group as aggregated circuit breaker(we defines it as Aggregated_CB : A_CB) in order to reduce the searching space and to enhance the efficiency of the representation of connection structure and the topology identification.

In this paper, the predicate of aggregated circuit breaker is defined as follows.

a_cb(CB_name, DS_name_list, ES_name_list, status)

Here, 'CB_name' describes the name of circuit breaker and 'DS_name_list' describes a set of disconnecting switch connected to circuit breaker. 'ES_name_list' describes a set of ground switch. 'status' describes ON/OFF status of aggregated circuit breaker. 'status' is determined by the status of circuit breaker and disconnection switches.

PROLOG rules to determine the ON/OFF status of aggregated circuit breaker are as follows.

$$
\begin{aligned}
& \text { a_cb(CB_name,[DS_name1,DS_name2], ,,'on) } \\
& \therefore \text { - cb(CB_name, "on"),ds(DS_name1, "on"), } \\
& d s\left(D S \_\right. \text {name2, "on”). } \\
& \text { a_cb(CB_name,_,, "off }):-c b\left(C B \_n a m e, " o f f\right) \text {. } \\
& \text { a_cb(CB_name,[DS_name1,],_, "off) } \\
& \text { :-,ds(DS_name1, "off"'), } \\
& \text { a_cb(CB_name, [_,DS_name2], ,' off }) \\
& \therefore \text {-,ds(DS_name2, "off”'), }
\end{aligned}
$$

Figure 3 shows the structure of simplified substation using the aggregated circuit breaker. As shown in figure 3, about 100 switches reduced to 22 Aggregated_CBs. 
As a result of a new definition, more complex connection structure of figure 1 can be changed into a simple connection structure of figure 3. If aggregated circuit breaker is used, the connection structure of substation can be represented very simple and the time of topology identification can be reduced remarkably. Also, the proposed aggregated circuit breaker can contribute to improving performance of all the developed

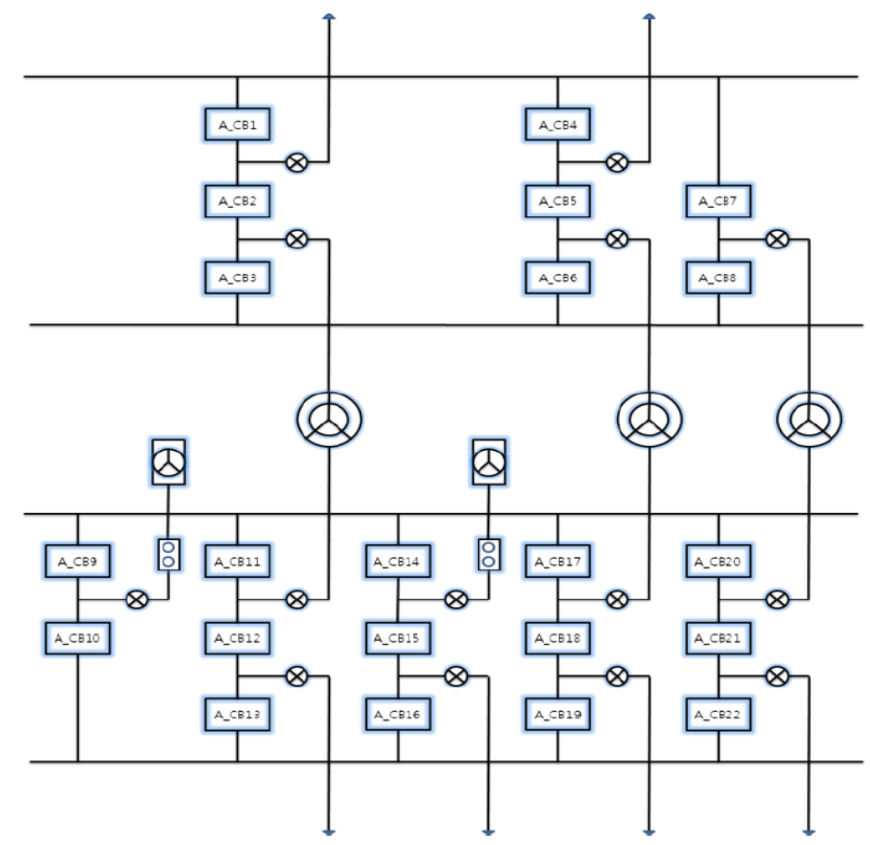

Fig. 3 Structure of aggregated substation

intelligent system for $765 \mathrm{kV}$ substation.

\section{DATA REPRESENTATION}

\section{A. Definition of unit device}

Data representation is closely related with proposed topology processor. In this paper, unit devices and switches are described. The predicates of unit devices and switches are as follows.

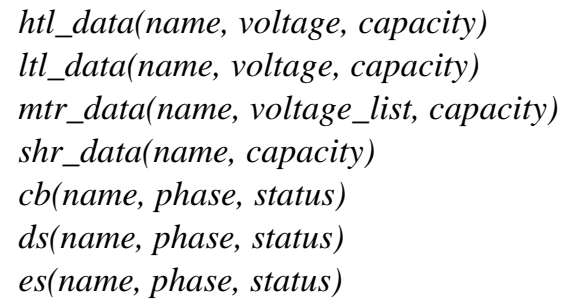

Here, 'name' describes the name of each unit device and 'voltage' describes the voltage of each unit device. 'capacity' describes the capacity of each unit device and 'voltage_list' in predicate $\mathrm{mtr}$ is a set of voltage connected to transformer. 'status' is ON or OFF status of switches.

\section{B. Definition of connection structure between unit devices}

As shown in figure 3 , this paper changed the complex connection structure of $765 \mathrm{kV}$ substation to simple connection

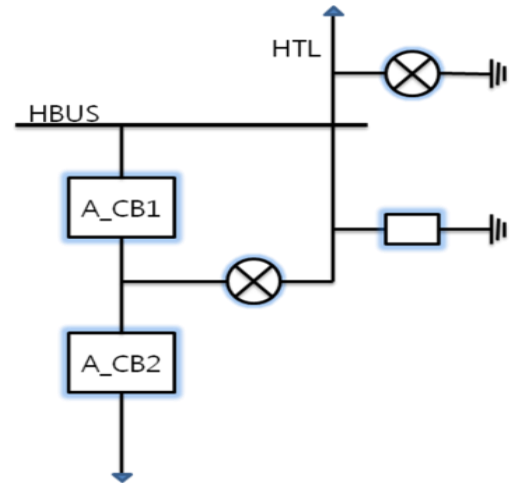

Fig. 4 Connection structure between $765 \mathrm{kV}$ TL and bus

structure using the aggregated circuit breaker. This paper described the connection structure of the simple substation in figure 3 as follows.

Connection structure between $765 \mathrm{kV}$ transmission line and $765 \mathrm{kV}$ bus shows in figure 4 .

As shown in figure 4, transmission line is connected to bus through disconnecting switch and Aggregated_CB. The predicate of connection structure between $765 \mathrm{kV}$ transmission line and bus is as follows.

tl_hbus_dat(tl_name, ds_name, a_cb_list)

Here, 'ds_name' describes the disconnecting switch connected to transmission line. 'a_cb_list' describes a set of aggregated circuit breaker connected to $765 \mathrm{kV}$ bus.

Connection structure between $345 \mathrm{kV}$ transmission line and

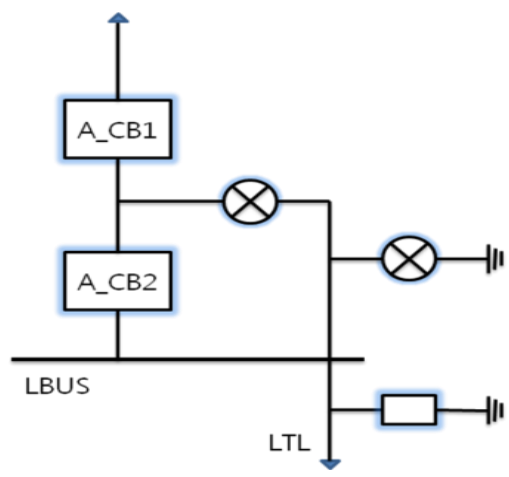

Fig. 5 Connection structure between $345 \mathrm{kV}$ TL and bus

$765 \mathrm{kV}$ bus shows in figure 5 .

The predicate of connection structure between $345 \mathrm{kV}$ transmission line and bus is as follows.

lbus_tl_dat(tl_name,ds_name, a_cb_list)

Here, 'ds_name' describes the disconnecting switch connected to transmission line. 'a_cb_list' describes a set of aggregated circuit breaker connected to $345 \mathrm{kV}$ bus. The structure of $765 \mathrm{kV}$ and $345 \mathrm{kV}$ bus shows in figure 6 .

The predicates of $765 \mathrm{kV}$ and $345 \mathrm{kV}$ bus are as follows. 


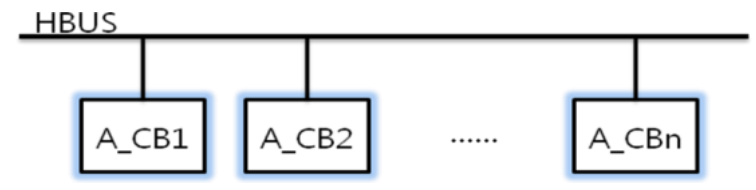

Fig. 6 Structure of bus

口 hbus_daa(hbus_name, a_cb_list)

口 lbus_daa(lbus_name, a_cb_list)

Here, 'a_cb_list' describes a set of aggregated circuit breaker connected to bus.

Connection structure between $765 \mathrm{kV}$ bus and transformer

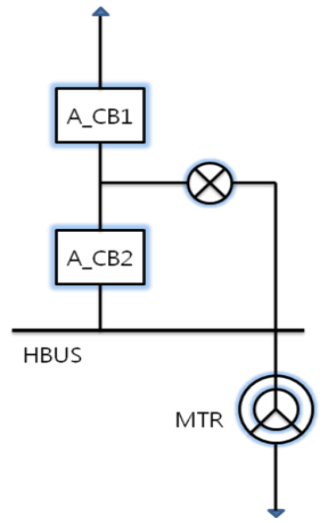

Fig. 7 Connection structure between $765 \mathrm{kV}$ bus and MTR

shows in figure 7.

The predicate of connection structure between $765 \mathrm{kV}$ bus and transformer is as follows.

hbus_mtr_dat(tr_name, ds_name, a_cb_list)

Here, 'tr_name' describes name of transformer, ,ds_name describes disconnecting switch connected to transformer and „a_cb_list ${ }^{\text {ec }}$ describes a set of aggregated circuit breaker connected to transformer.

Connection structure between $345 \mathrm{kV}$ bus and transformer shows in figure 8.

The predicate of connection structure between $345 \mathrm{kV}$ bus and transformer is as follows.

mtr_lbus_dat(tr_name, ds_name, a_cb_list)

Here, 'tr_name' describes name of transformer, ,ds_name ${ }^{\text {ee }}$ describes disconnecting switch connected to transformer and ,a_cb_list ${ }^{\text {"e }}$ describes a set of aggregated circuit breaker connected to transformer.

\section{TOPOLOGY IDENTIFICATION}

The above-mentioned definition just describes unit device and all possible connection structure of $765 \mathrm{kV}$ substation, but

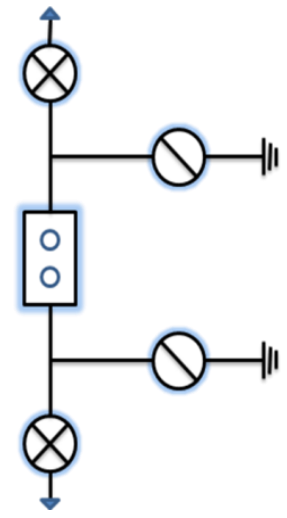

Fig. 8 Connection structure between $345 \mathrm{kV}$ bus and MTR

can "t describe real-time topology yet. The real-time topology must be determined by the inference process using the status data of switch such as circuit breaker and disconnecting switch.

The proposed topology processor identifies topology through the inference process classified into two steps. The first step is determination of the status of aggregated circuit breaker. The second step is topology identification using the aggregated circuit breaker. In the first step, topology processor uses the above-mentioned rules and the status data of switch such as circuit breaker, disconnecting switch and ground switch. In the second step, topology processor uses the duplex searching method. Figure 9 shows duplex searching method including all possible topology of $765 \mathrm{kV}$ substation.

As shown in figure 9, the upper side displays the above-mentioned connection structure between unit devices including aggregated circuit breakers. The lower side shows the intersection between aggregated circuit breakers included at each unit device. Therefore, the real-time topology can be identified through the search of intersection between aggregated circuit breakers. The following predicates describe the real-time topology of $765 \mathrm{kV}$ substation and are generated by the result of

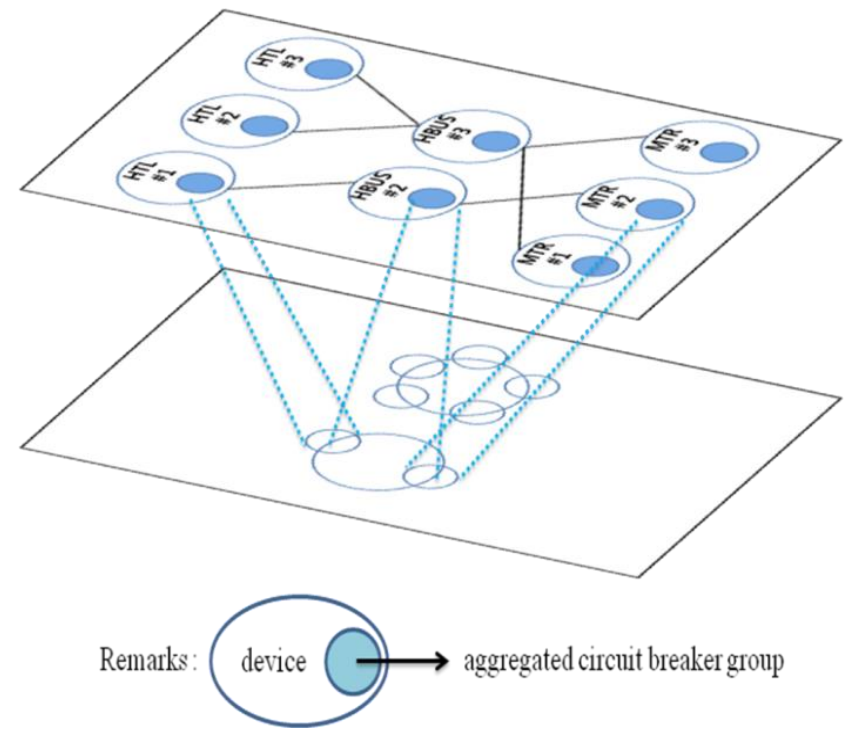

Fig. 9 Duplex searching method 
the inference process.

htl(name, status)

ltl(name, status)

hbus(name, status, tl_name_list, tr_name_list)

mtr(name, status, hbus_name, lbus_name)

lbus(name, status, tr_name, tl_name_list)

Here, 'name' describes name of each unit device and 'status' describes 'energized' or 'de-energized' of each unit device. 'tl_name_list' and 'tr_name_list' are sets of devices connected to each unit device These predicates are the output of the proposed topology processor and are used to database of the intelligent system.

\section{THE RESULT OF TOPOLOGY PROCESSOR}

The topology processor is used to identify the real-time topology of substation. The topology processor generates the predicates which is expressing the topology structure. The generated predicates are not presented to operator and are stored in database of intelligent system.

The predicates which the topology processor generates are as follows.

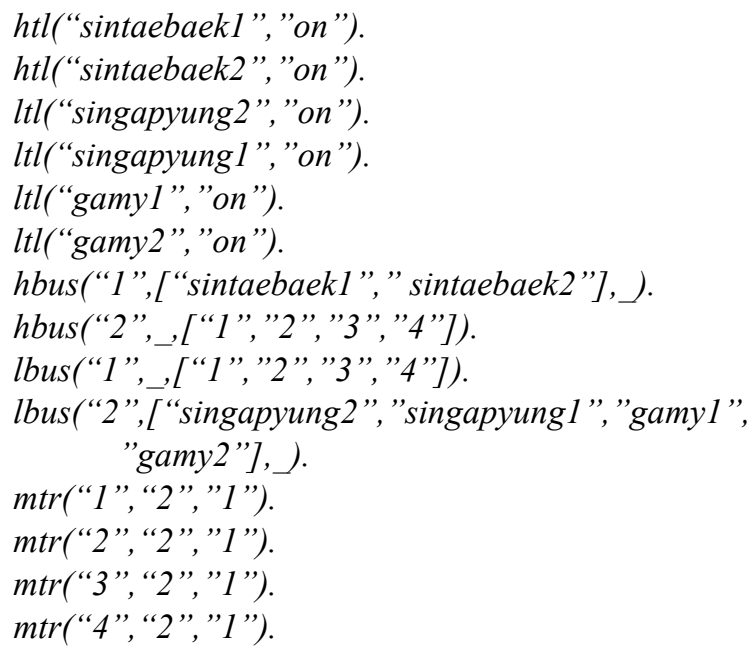

\section{CONCULUSION}

This paper proposed the topology processor to identify topology of double-bus $1.5 \mathrm{CB}$ structured $765 \mathrm{kV}$ substation in Korea. The proposed topology processor redefines switching group as one aggregated circuit breaker and remarkably reduces the searching space and searching time of topology identification. This paper defines connection structure to identification of simplified $765 \mathrm{kV}$ substation.

The proposed topology processor is developed using the data of a practical $765 \mathrm{kV}$ substation in Korea. The topology processor is indispensable component for intelligent substation automation area, and will improve the performance of various intelligent systems.

\section{ACKNOWLEDGMENT}

This work has been supported by Korea Institute of Energy
Technology Evaluation and Planning (KETEP) and the Ministry of Trade, Industry \& Energy (MOTIE) of the Republic of Korea (No. 20174010201620), and the National Research Foundation of Korea (NRF) grant funded by the Korean government (MSIT) (No. 2020R1F1A1076667).

\section{REFERENCES}

[1] Shunichi Ito, Isao Hata, Taizo Hasegawa et. al., "Advanced Operation Guidance Expert System for 500kV Substation,” pp. 405-412, Third Symposium on ESAP, 1991, Japan.

[2] S.A.R. Piereti, A.C.B. Delbem, J.B.A. London, "Tracking Network Topology Processor Using Node-Depth Representation," PowerTech 2007, pp. 143-148, 2007.

[3] Y. He, D. Yu, et. al., "An Efficient Topology Processor for Distribution Systems," IEEE Transaction on Power System, pp. 824-829, 2001.

[4] P.D. Yehsakul, I. Dabbaghchi, "A Topology-Based Algorithm for Tracking Network Connectivity," IEEE Transaction on Power Systems, Vol. 10, No. 1, 1995

[5] H.J. Lee, Y.M. Park, "A Restoration Aid Expert System for Distribution Substations," IEEE Transaction on PWRD, Vol. 8, No. 4, pp. 1765-1770, 1996.

[6] H.J. Lee, et. al., "Dealing Uncertainties in the Fault Diagnosis of Power System," Engineering Intelligent Systems, Vol. 7, No. 4, pp. 168-176, 1999.

[7] H.J. Lee, C.H. Lim, et. al., "Identification and Correction of Topology Errors in Distribution Substations," ICIC Express Letters, Vol. 3, No. 4, pp. 1321-1326, 2009.

[8] N. Singh, H. Glavitsch, "Detection and Identification of Topological Errors in Online Power System Analysis," IEEE Transaction on Power Systems, Vol. 6, No. 1, pp. 324-331, 1991.

[9] M. Prais, A. Bose, "A Topology Processor that Tracks Network Modifications Over Time," IEEE Transaction on Power Systems, Vol. 3, No. 3, pp. 992-998, 1988.

\section{Contribution of individual authors to the creation of a scientific article (ghostwriting policy)}

Conceptualization, H.L. and W.Y.; methodology, W.Y; validation, M.Y. and J.O.; writing - original draft preparation, H.L.; writing - review and editing, J.O.; project administration, H.L.; funding acquisition, H.L. All authors have read and agreed to the published version of the manuscript.

\section{Sources of funding for research presented in a} scientific article or scientific article itself

This work was supported by the Korea Institute of Energy Technology Evaluation and Planning (KETEP) and the Ministry of Trade, Industry \& Energy (MOTIE) of the Republic of Korea (No. 20174010201620).

\section{Creative Commons Attribution License 4.0 (Attribution 4.0 International , CC BY 4.0)}

This article is published under the terms of the Creative Commons Attribution License 4.0

https://creativecommons.org/licenses/by/4.0/deed.en_US 